\title{
Multiple-address Technique of Satellite Communication
}

\author{
Weijun $\mathrm{Yu}^{\mathrm{a}, *}$, Xianyi Qian ${ }^{\mathrm{b}}$ \\ ${ }^{a}$ Changzhou Institute of Technology, CZU, Changzhou, China \\ ${ }^{b}$ School of Electronic Information \& Electric Engineering, Changzhou Institute of \\ Technology,CZU,Changzhou, China
}

\begin{abstract}
In this paper, we have studied the operation modes of satellite communication system. They include that signal transmission mode, signal processing mode and signal exchanging mode. We have especially studied the multiplex mode, modulation mode, coding mode, multiple-address connection, and channel distribution and exchanging system. All we have studied are important to the satellite communication station's changing and signal processing.
\end{abstract}

Index Terms: Multiple-address; Modulation; Coding; Synchronization of TDMA system

(C) 2011 Published by MECS Publisher. Selection and/or peer review under responsibility of the Research Association of Modern Education and Computer Science.

\section{Multiple-address connection}

Multiple-address connection is the basic characteristic of satellite communication. It is the main content of satellite communication system. The so-called satellite communication system is the operation mode of satellite communication system, which is the signal transmission mode, signal processing mode and signal exchanging mode. They include multiple-address mode, modulating mode, coding mode and multiple-address connection mode and channel distribution and exchanging system.

Multiple-address connection means that many earth stations build their own channels by means of the common satellites, so this has realized a kind of communication mode between many earth stations. Multipleaddress technique has improved the coefficient utilization of satellite communication link and flexibility of communication connection. [1]

Although multiple-address is different from multiplexing, they are similar in something. They are both used to study and resolve channel's multiplexing, such as how to distinguish the combined signal during the process of transmission. They both include three steps during the process of communication: combining many

\footnotetext{
* Corresponding author.

| E-mail address: hbxfqxyqxy_123@163.com
} 
signals, transmission of the combined signal and separating the combined signal. Fig. 1 shows the three steps. On the other hand, the signals' multiplexing and separating in multiplexing are based on base band, and the signals come from speech channel directly. While the signals which processed in multiple-address come from different earth stations.[2]

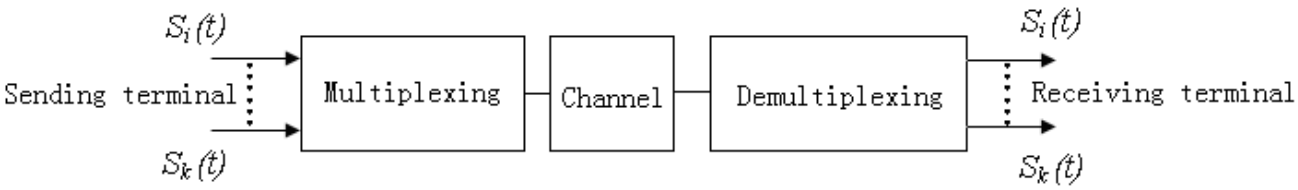

Fig. 1. Multiplexing and demultiplexing of signals

So, we should distinguish signal and address clearly. When one earth station only sends one radio carrier or one radio frame, the concept of multiple-address is clear. But when one earth station sends several radio carriers or radio frames, we need to consider how to distinguish the concrete radio carriers or frames. So, there are some differences between distinguishing signal and distinguishing address. So, multiple-address connection is sometimes called multi-join.

The basis of realizing multiple-address technique is splitting signal, which is designed in signal sending terminal. So, many earth stations in the whole system send different signals. And these earth stations' receiving terminals should have the ability of recognizing signal. This means that every receiving terminal can acquire the needed signal. Fig.2 shows the whole system.

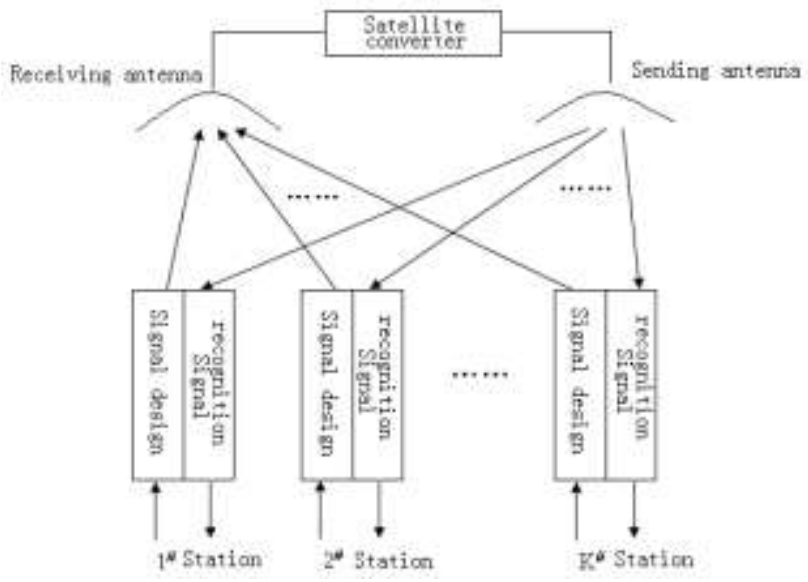

Fig. 2. Realization of multiple-address connection

A radio electric signal can be represented by many parameters. The basic parameters consist of radio frequency, time and space. The difference between signals can be concentrated on the difference between signal parameters.

In satellite communication, signal distribution and recognition can be realized by any parameter of signal. The most effect method which used to realize multiple-address connection is orthogonality if we consider the affection of noise and other factors.[3]

Usually, the design of good multiple-address system is a very complicated work, and we should consider the following factors: capacity requirements, coefficient of utilization of satellite frequency band, utilization of satellite power, adaptation to the increment of business and network, ability to processing all kinds of different 
businesses, technique factor and economic factor. There are many kinds of technologies to realize multipleaddress connection mode. The usual multiple-address mode includes FDMA, TDMA, CDMA and SDMA at present. On the other hand, it also includes frequency reuse technology which using cross polarization distribution multiple-address connection mode. Because of the combination of computer and communication, multiple-address technology is still being developed.

Multiple-address technology is not only applied in satellite communication, but also ground communication net. Many communication stations communicate each other by means of one radio-frequency channel in ground communication net; they also need multiple-address connection technology. Such as mobile communication, spread spectrum communication and microwave communication. [4]

\section{TDMA}

TDMA is a kind of digital multiple-address technology. It makes satellite receive every earth station terminal's signal in the separated slot time which is called Burst. This kind of technology can avoid intermodulation products which caused by repeaters' nonlinearity in FDMA. Ground terminal must have certain time and range of satellite system, which makes the sent signal reach satellite correctly in the prescribed slot time. Fig. 3 shows the typical TDMA net structure. In this kind of net structure, every high bitrate Burst signal (such as orthogonal PSK modulation) can reach satellite in the prescribed slot time. Because only one signal can enter satellite repeater at any slot time, there isn't any intermodulation products which caused by instant nonlinearity. We should notice that the bit-rate of transmitting signal usually is higher than ground terminal's continuous input bit-rate. Comparing with FDMA system, TDMA has the following characteristics:

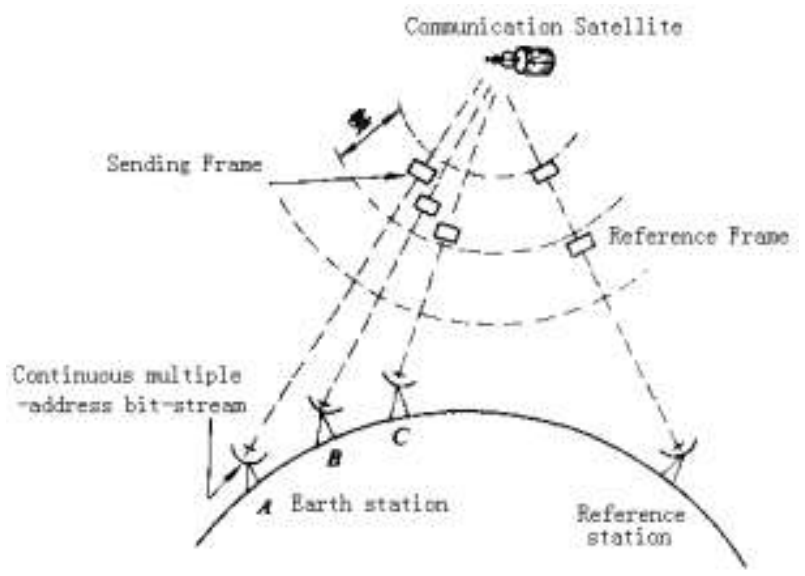

Fig. 3. TDMA net structure

- Satellite repeater can operate near the saturation point, so it can use satellite power effectively.

- In TDMA, the capacity can't be decreased which the net stations' number adding. After accepting DSI technology, the transmission capacity has been added almost one time. One INTELSAT-V satellite repeater which operates at $80 \mathrm{MHz}$ can provide 1600 pieces of $64 \mathrm{~kb} / \mathrm{s}$ speech channels without accepting DSI technology. It can provide 3200 speech channels whose frequency is $64 \mathrm{~kb} / \mathrm{s}$ after having accepted DSI technology.

- We can add new business or change the present business by changing the length and position of Burst.[5] 


\section{System structure}

Every TDMA earth station has digital bit stream which is parallel input or analog signal stream which has been digitalized. Fig. 4 and Fig.5 show that these signals have been send every earth station. These sent signals are located in the TDMA frame. TDMA receiver demodulates the TDMA frame which from sending terminal and multiple-address multiplexer, and then divide these signals into serial digital signal stream which are independent each other. The TDMA signal consists of a group of Burst signals which from earth station. Fig.5 shows that there is a small slot time in every two Burst signals, which is called protecting time. It makes the interfacing two Burst pulses be not overlapped each other. The first Burst signal in TDMA frame doesn't have any business information, and it is only used to control net and synchronization, which is called Reference Burst signal. The period of TDMA frame is the integral multiple of $125 \mu \mathrm{s}$, which is corresponding to the frequency of $8 \mathrm{kHz}$. Every Burst signal is sent as the period of TDMA frame.

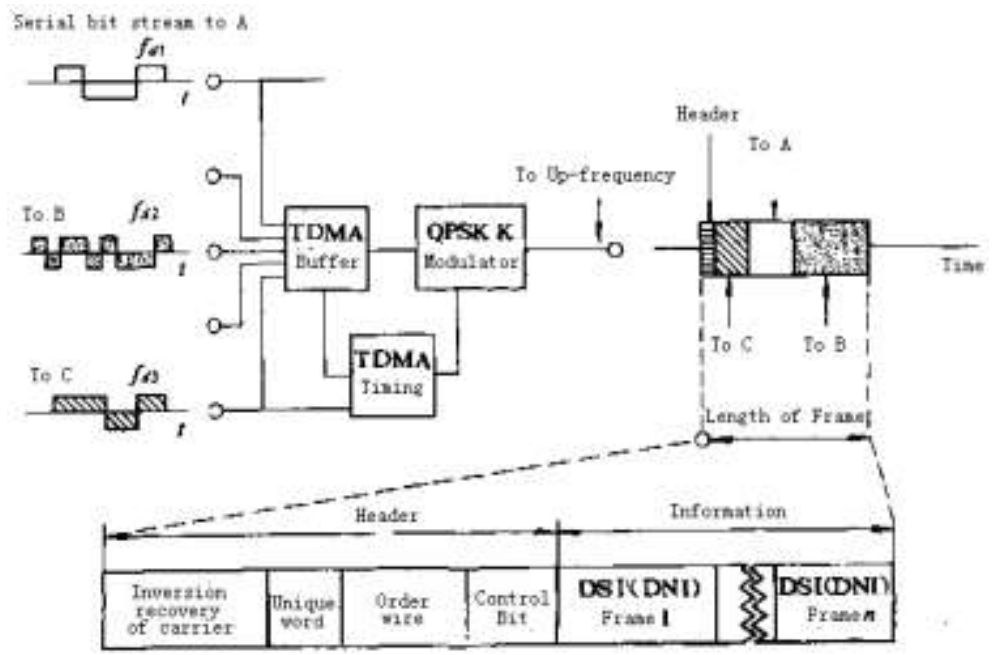

Fig. 4. Sending TDMA data

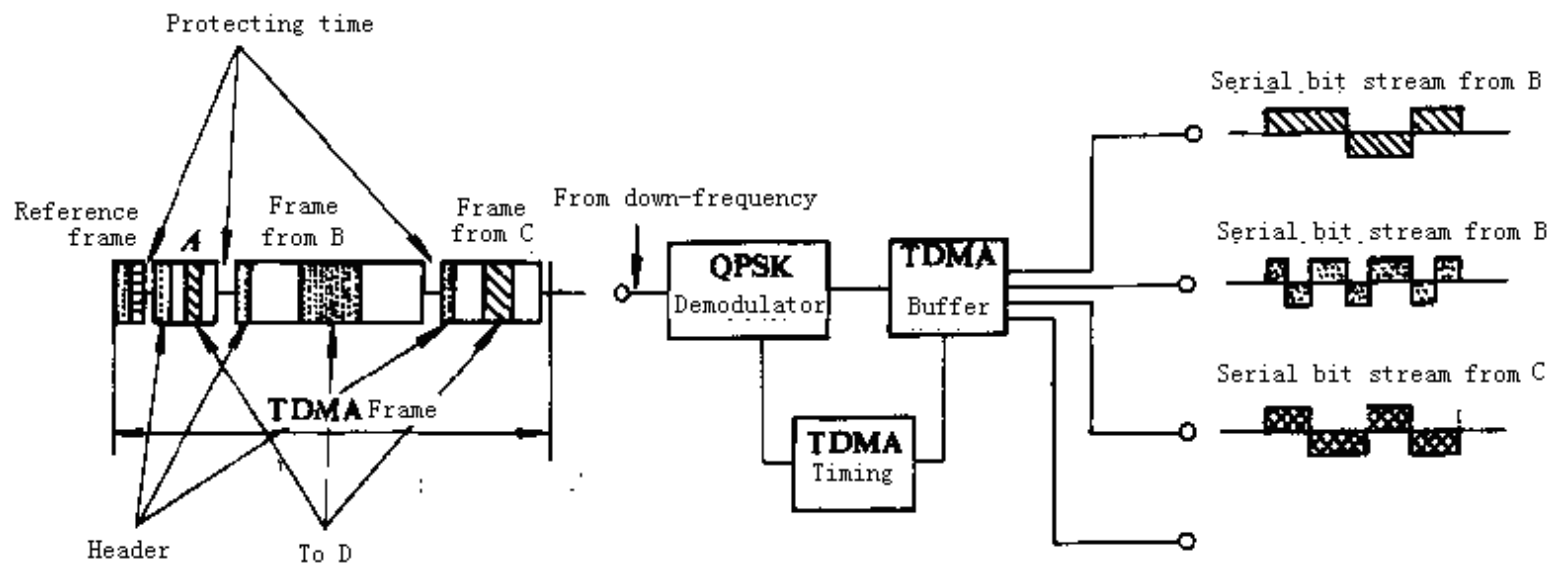

Fig. 5. Receiving data of TDMA 
Fig.4 is the format of business frame. In one TDMA frame, information bit stream are be distributed groups, which are called Burst signal. Every Burst signal includes the information which from someone interface module to another corresponding earth station information. In front of information bit, there is a group of bits, which is called header. It is used to control synchronization and net. The reference frame only consists of header, and the ordinary frame header includes the following parts:[6]

- Bit timing of inversion recovery and carrier. It can provide the needed carrier reference and location timing timer which used in receiving terminal.

- Unique word. It is used to recognize TDMA frame's starting position and every bit's position.

- Order wire bit word. It is used to transmit work phone and information via telex.

- Control word. It is used to transmit net management information.

\section{Synchronization of TDMA system}

TDMA system is about several different synchronization problems.

When demodulate Burst PSK carrier, we must recover carrier and bit timing in starting part's inversion recovery of every frame. So, the usual TDMA demodulator uses rapid circuit to recover carrier and timing signal. [7]

We should time the every Burst signal. This kind of synchronization can prevent frames which from all earth stations to be overlapped in satellite repeater, which is called frame synchronization. Frame synchronization can make every pulse enter satellite repeater with respect to reference pulse keeping the prescribed timing difference. We have studied the following several methods which aiming at the frame synchronization:

- Synchronization of global beam: Every transmitting station acquires the timing error by checking the received signal sequence.

- Synchronization of feedback: After having checked the timing error, the receiving station or reference station sends the frame position error information to sending station by means of control channel.

- Synchronization of open loop: Confirming transmitting timing by computing or measuring the distance from every station to satellite.

We should notice that the synchronization of frame is on the basis of close loop mode. These synchronizations require that every station can receive the sent frame.

On the other hand, if the TDMA-SS used in the system, the down transmission should use point beam. So it must use others synchronization modes. Because every Burst signal must send in accordance with different address using different beam which can be realized by satellite switching matrix. TDMA frame uses a kind of synchronous switching sequence, and we can transmit information by using one or more fixed slot time.

\section{Conclusions}

In this paper, we have discussed modulation technology, multiple-address technology, coding technology, signal processing technology, satellite communication net, satellite communication link and TV broadcast by satellite, which are all used in satellite communication. What we have studied has great significance to the satellite ground station's maintenance and adjusting.

\section{References}

[1] TIAN Bin, CHEN Wen-han, Vehicle Synchronous Orbiting Satellite Mobile Communication System[J], INFORMATION AND ELECTRONIC ENGINEERING, 2005 3(2) 
[2] WANG Wei-min, Cross Polarization Problem in Frequency Re-use Communication System[J], JOURNAL OF MICROWAVES, 2005 21(z1)

[3] Liu Qianlin, Chen Dagang, Design and Implementation of Simulation Training Software for VSAT[J], WIRELESS COMMUNICATION TECHNOLOGY, 2000 9(1)

[4] Tu Chongyang, A method for the ship-board antenna to track satellites[J], JOURNAL OF XIDIAN UNIVERSITY, 2002 29(2)

[5] Wang Shilu, A Study of New Technologies in Satellite Mobile Communications[J], JOURNAL OF NANJING UNIVERSITY OF POSTS AND TELECOMMUNICATIONS(NATURAL SCIENCE), 2002 22(4) [6] SHAO Shu-yuan, ZHANG Yang, Estimation of Interference Delay in PCMA System[J], INFORMATION AND ELECTRONIC ENGINEERING, 2006 4(1)

[7] FU Di, GAO Yong, Signal Interception Method for Asymmetric PCMA Satellite Communication Systems[J], MODERN ELECTRONICS TECHNIQUE, 2007 30(7) 